\title{
CORRIGENDA
}

\section{Links between plant and fungal communities across a deforestation chronosequence in the Amazon rainforest}

Rebecca C Mueller, Fabiana S Paula, Babur S Mirza, Jorge LM Rodrigues, Klaus Nüsslein and Brendan JM Bohannan

The ISME Journal (2014) 8, 1551; doi:10.1038/ismej.2014.62

Correction to: The ISME Journal (2014) 8, 1548-1550; doi:10.1038/ismej.2013.253; published online 23 January 2014

Since the publication of this article, the authors have identified errors in the Supplementary Information, mainly concerning the listed primer sequences.

There was a deletion in the primer sequence listed for the second stage PCR. The erroneous sequence was 5'-AAGCAGAAGACGGCATACGAGATCGGTCT GGCATTCCTGC-3' ${ }^{\prime}$, but the correct primer sequence is $5^{\prime}$-AAGCAGAAGACGGCATACGAGATCGGTCTC GGCATTCCTGC- $3^{\prime}$.
In addition, the sequence listed for ITS2 was incorrectly listed as the sequence for ITS2-Melamspora. The correct sequence for the ITS2 primer used in this study is $5^{\prime}$-GCTGCGTTCTTCATCGA TGC- $3^{\prime}$.

Finally, the concentration of the dNTPs is listed at $\mu \mathrm{M}$, but should be in mM.

The errors have now been rectified, and the correct Supplementary Information now appears on the online version of the article. In addition, it also accompanies the online version of this corrigendum.

The authors apologise for any inconvenience caused.

\section{Classification and quantification of bacteriophage taxa in human gut metagenomes}

Alison S Waller, Takuji Yamada, David M Kristensen, Jens Roat Kultima, Shinichi Sunagawa, Eugene V Koonin and Peer Bork

The ISME Journal (2014) 8, 1551-1552; doi:10.1038/ismej.2014.66

Correction to: The ISME Journal (2014) 8, 1391-1402; doi:10.1038/ismej.2014.30; published online 13 March 2014

Since the publication of this article, the authors have identified an error in Figure 1a, namely that the columns were not clustered and the column labels were incorrect, in that 252.Gut.PFPR and 61.Gut. Virome were reversed.
The correct figure is shown here.

The error has now been rectified, and the correct article appears in this issue. The html and online pdf versions have also been rectified, and now carry the correct paper.

The Authors would like to apologize for any inconvenience this may have caused. 

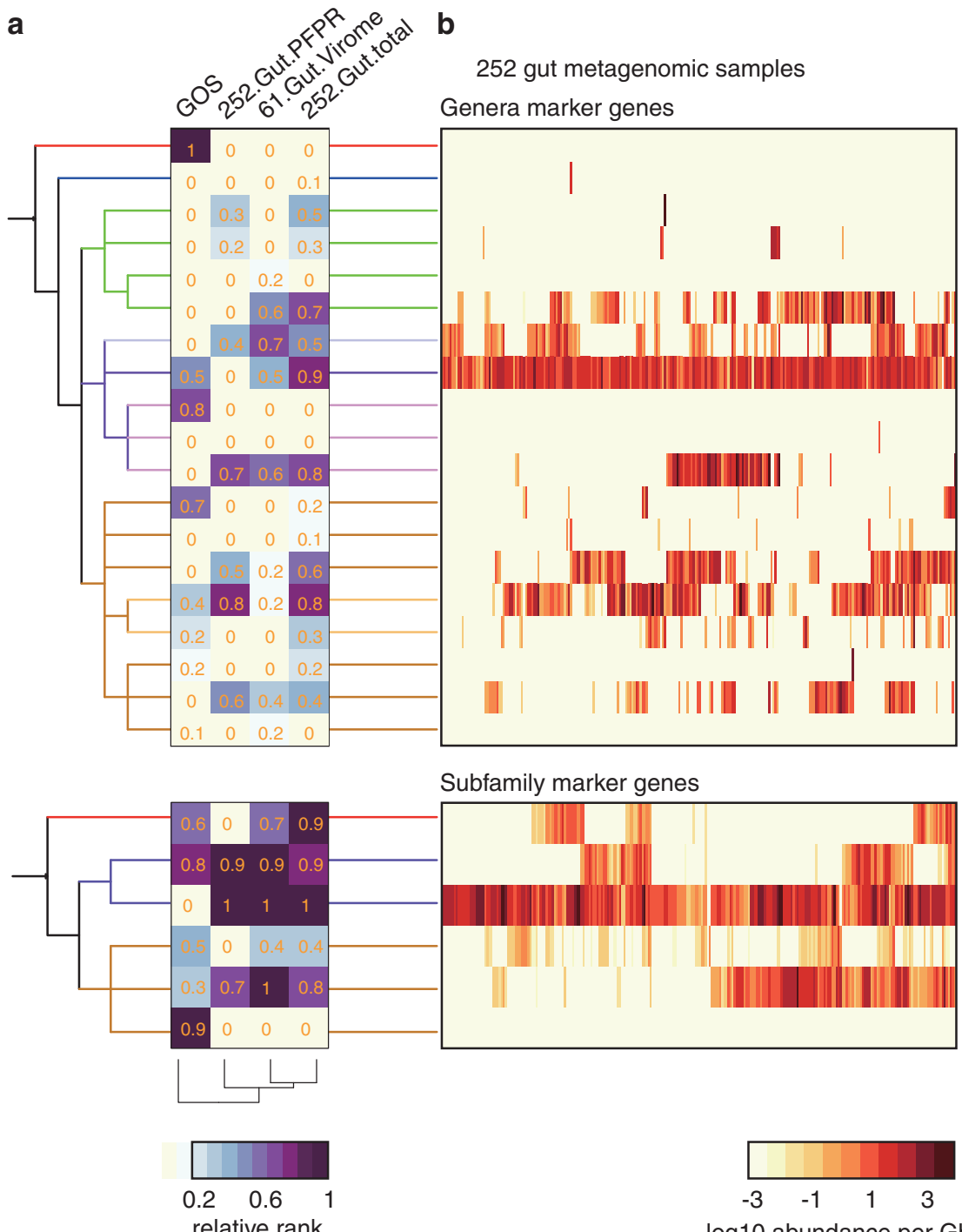

\section{Cladogram Branch Colors \\ $\square$ Microviridae $\square$ Myoviridae \\ $\square$ Tectiviridae $\square$ Picovirinae \\ $\square$ Siphoviridae $\square$ Autographvirinae \\ $\square$ Podoviridae $\square$ Peduovirinae}

Subfamily marker genes

Microvirus

Tectivirus

T1-like viruses

Lambda-like viruses (rcll 17\%)

Lactococcus phage ul36 ( $\mathrm{rcll}$ 67\%)

936 group of lactococcal phages

Phi29-like viruses

N4-like viruses

unclass.Autographivirinae.1132574

SP6-like viruses

T7-like viruses

I3-like viruses

PhiKZ-like viruses

$\mathrm{Mu}$-like viruses (rcll 50\%)

Hp1likevirus

P2-like viruses

PB1likevirus

PhiCD119likevirus

FelixO1likevirus

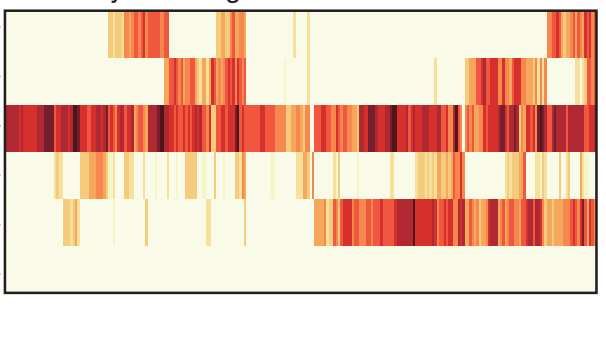

\section{Gokushovirinae}

Autographivirinae

Picovirinae

Peduovirinae

Spounavirinae

Tevenvirinae

Figure 1 Taxonomic classification and quantification of phages in the human gut and the Oceans. (a) Relative rank of phage taxa in the human gut and the Ocean. The abundance of each taxon was used to determine its rank, and the relative rank is then the rank divided by the maximum rank in that sample set. Thereby, a relative rank of 1 is the most abundant taxon in that sample. Each column represents one of the four different sample sets: GOS (82 samples from the Global Ocean Sampling Expedition), 252.Gut.PFPR (only the subset of prophage-encoded genes in 252 gut metagenome samples), 61.Gut.Virome (all of the genes with 61 samples from three published studies of virus-enriched gut metagenomes), 252.Gut.total (all of the genes contained within 252 gut metagenome samples). The columns are clustered by similarity, as illustrated by the dendrogram below the columns. (b) Abundance of each phage taxon within the 252 human gut samples (total metagenomic genes). These abundances were derived by dividing the length-normalized base coverages by the total gene abundance in each sample, and multiplied by $10^{9}$ to yield the abundance per Gbp. For those taxa for which marker genes are not quantitative (see Materials and methods), the recall is indicated in brackets. 